\title{
JUST PUBLISHED
}

\section{THE PRINCIPLES}

\section{OF INTERNATIONAL HUMANITARIAN LAW ${ }^{1}$}

\author{
by \\ Jean Pictet \\ Director-General, International \\ Committee of the Red Cross \\ Lecturer at Geneva University
}

In this sixty-page book the writer defines fully and with concision humanitarian law in its widest sense, the laws of war of The Hague and Geneva, and the principles which form the basis for this humanitarian law.

This clear summary is understandable to everybody interested in humanitarian ideas and actions in the world today. In addition, the appendix is a chart of the principles of humanitarian law.

It will be recalled that an earlier work by this author, The Principles of the Red Cross, gives the general reader a clear exposition of its subject. Copies of this book, which has already had considerable success, are available in French, English, German and Spanish, from the ICRC Geneva, which published the book.

1 The Principles of International Humanitarian Law can be obtained from the ICRC, 7 avenue de la Paix, 1211 Geneva (postal cheque account No. 12-5527). Cost Sw.fr. 8.- 


\section{RECENT PUBLICATIONS}

Published by THE ICRC

Geneva Conventions of August 12, 1949. Essential provisions, 1965, 8vo, 3 pp. Sw. Fr. 0,50.-

Claude Pilloud. Reservations to the 1949 Geneva Conventions, $1965,8 v o, 8$ pp. Sw. Fr. 2.50

ICRC. Annual Report 1965. 1966, 8vo, 83 pp. Sw. Fr. 7.-

\section{Published by the Centenary Commission of the Red Cross in SwitzerLand}

World Conference of Educators (Lausanne, 19-23 August 1963), 1964, 8vo, 205 pp. Sw. Fr. 5.-

International Red Cross Meeting of First-Aiders, Macolin (Switzerland), 18-24 August 1963, 1963, 8vo, 55 pp. Sw. Fr. 3.-

The Red Cross and Philately (1863-1963), 1965, 8vo, 24 pp., Ill., Sw. Fr. 2.-

Seminar on the activity of the Red Cross on behalf of the victims of armed conflicts, Geneva, August 1963, 1965, 8vo, 238 pp., Ill., Sw. Fr. 7.-

Centenary Congress of the International Red Cross. Commemoration Day - Council of Delegates, Geneva 1963, Ill., 120 pp., Sw. Fr. 10.- 


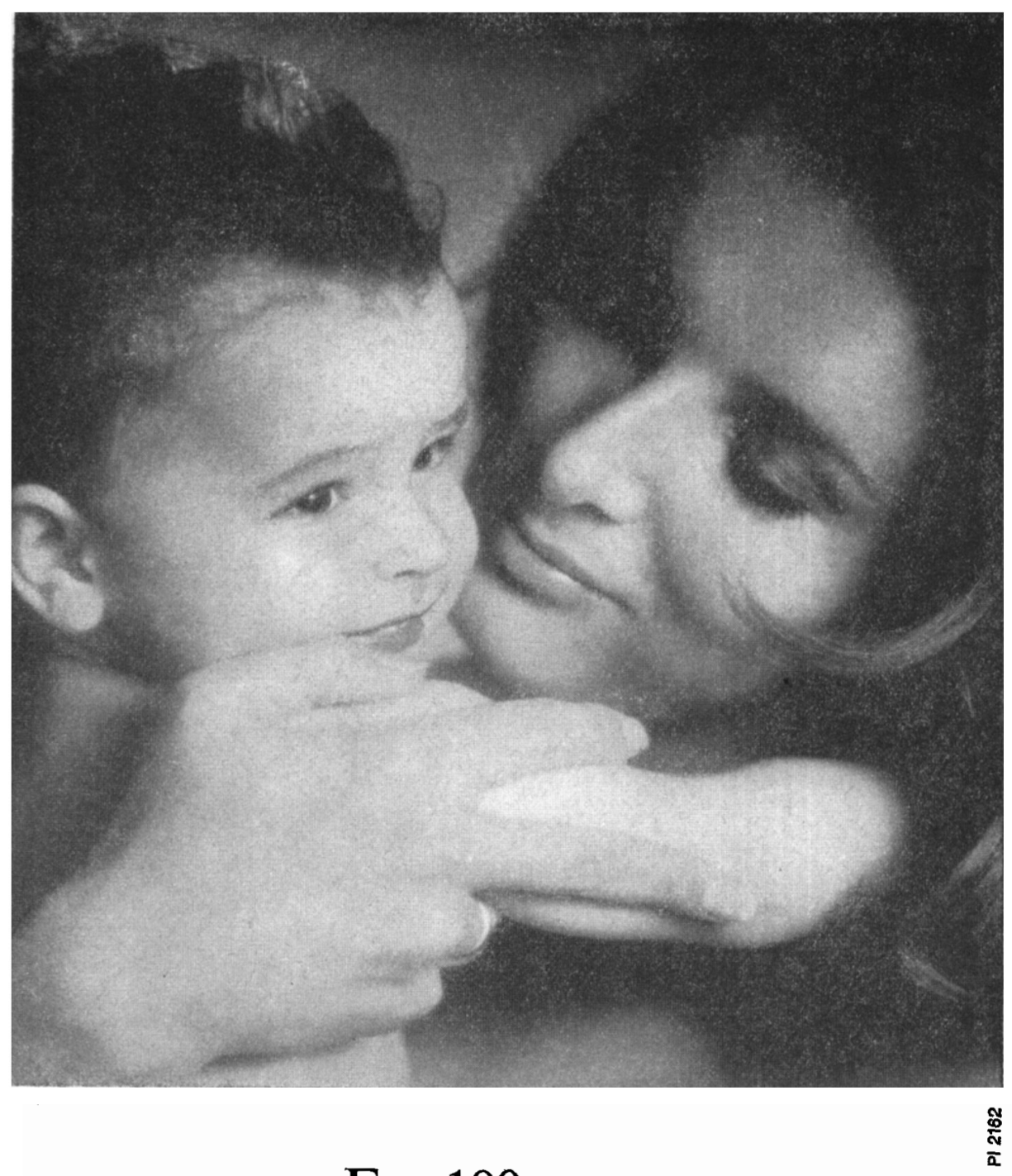

Nestlé has stood for service to childhood throughout the world.

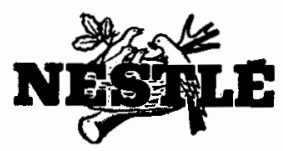



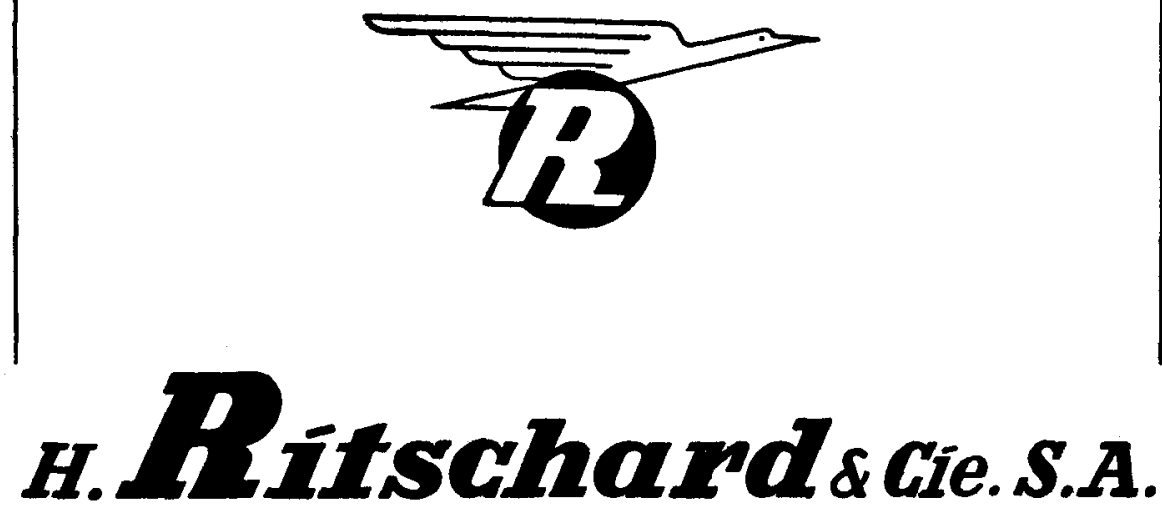

\section{INTERNATIONAL TRANSPORT TRAVEL AGENCY}

GENEVA, 18 Place Comavin

Telephone 323400 - Teleprinter 22167

Exchange - Tickets - Sea passages

Insurance - Customs Agency

Road haulage - Storage

Home delivery of air and rail tickets on request by telephone

Branches :

LAUSANNE - BASLE - ZURICH - ANNEMASSE (France) 


\section{ADDRESSES OF CENTRAL COMMITTEES}

AFGHANISTAN - Afghan Red Crescent, Kabul.

ALBANIA - Albanian Red Cross, 35, Rruga Barrikadavet, Tirana.

ALGERIA - Central Committee of the Algerian Red Crescent Society, 15 bis Boulevard Mohamed V, Algiers.

ARGENTINE - Argentine Red Cross, H. Yrigoyen 2068, Buenos Aires.

AUSTRALIA - Australian Red Cross, 122-128 Flinders Street, Melbourne, C. 1 .

AUSTRIA - Austrian Red Cross, 3 Gusshausstrasse, Postfach 39, Vienna IV.

BELGIUM - Belgian Red Cross, 98, Chaussée de Vleurgat, Brussels 5.

BOLIVIA - Bolivian Red Cross, Avenida Simon-Bolivar, 1515 (Casilla 741), La Paz.

BRAZIL - Brazilian Red Cross, Praça da Cruz Vermelha 10-12, Rio de Janeiro.

BULGARIA - Bulgarian Red Cross, 1, Boul. S.S. Viruzov, Sofia.

BURMA - Burma Red Cross, 42, Strand Road, Red Cross Building, Rangoon.

BURUNDI - Red Cross Society of Burundi, rue du Marché 3, P.O. Box 1324, Bujumbura.

CAMBODIA - Cambodian Red Cross, $17 \mathrm{R}$ Vithei Croix-Rouge, P.O.B. 94, Phnom-Penh.

CAMEROON - Central Committee of the Cameroon Red Cross Society, rue HenryDunant, P.O.B. 631, Yaoundé.

CANADA - Canadian Red Cross, 95, Wellesley Street East, Toronto 5.

CEYLON - Ceylon Red Cross, 106 Dharmapala Mawatte, Colombo VII.

CHILE - Chilean Red Cross, Avenida Santa Maria 0150, Casilla 246 V., Santiago de Chile.

CHINA - Red Cross Society of China, 22 Kanmien Hutung, Peking, $E$.

COLOMBIA - Colombian Red Cross, Carrera 7a, 34-65 Apartado nacional 1110, Bogota D.E.

CONGO - Red Cross of the Congo, 24, Avenue Valcke, P.O. Box 1712, Kinshasa.

COSTA RICA - Costa Rican Red Cross, Calle 5a Apartado 1025, San José

CUBA - Cuban Red Cross, Calle Zulueta 471, Havana.

CZECHOSLOVAKIA - Czechoslovak Red Cross, Thunovska 18, Prague I

DAHOMEY - Red Cross Society of Dahomey, P.O. Box 1, Porto-Novo.

DENMARK - Danish Red Cross .Ny Vestergade 17, Copenhagen $K$.

DOMINICAN REPUBLIC - Dominican Red Cross, Calle Galvan 24, Apartado 1293, Santo Domingo.
ECUADOR - Ecuadorean Red Cross, Avenida Colombia y Elizalde 118, Quito.

ETHIOPIA - Ethiopian Red Cross, Red Cross Road No. 1, P.O. Box 195, Addis Ababa.

FINLAND - Finnish Red Cross, Tehtaankatu I A, Helsinki.

FRANCE - French Red Cross, 17, rue QuentinBauchart, Paris ( $8^{\mathrm{e}}$ ).

GERMANY (Dem. Republic) - German Red Cross in the German Democratic Republic, Kaitzerstrasse 2, Dresden A. 1.

GERMANY (Federal Republic) - German Red Cross in the Federal Republic of Germany, Friedrich-Ebert-Aliee 71, 5300 Bonn 1, Postfach (D.B.R.).

GHANA - Ghana Red Cross, P.O. Box 835, Acra.

GREAT BRITAIN - British Red Cross, 14 Grosvenor Crescent, London, S.W.1.

GREECE - Hellenic Red Cross, rue Lycavittou 1, Athens 135.

GUATEMALA - Guatemalan Red Cross, 3." Calle 8-40 zona 1, Guatemala C.A.

HAITI - Haiti Red Cқoss, rue Férou, Port-auPrince.

HONDURAS - Honduran Red Cross, Calle Henry Dunant 516, Tegucigalpa.

HUNGARY - Hungarian Red Cross, Arany Janos utca 31, Budapest $V$.

ICELAND - Icelandic Red Cross, Ølduggøtu 4, Reykjavik, Post Box 872.

INDIA - Indian Red Cross, 1 Red Cross Road, New Delhi 1.

INDONESIA - Indonesian Red Cross, Tanah Abang Barat 66, P.O. Box 2009, Djakarta.

IRAN - Iranian Red Lion and Sun Society, Avenue Ark, Teheran.

IRAQ - Iraqi Red Crescent, Al-Mansour, Baghdad.

IRELAND - Irish Red Cross, 16 Merrion Square, Dublin 2.

ITALY - Italian Red Cross, 12, via Toscana, Rome.

IVORY COAST - Ivory Coast Red Cross Society, B.P. 1244, Abidjan.

JAMAICA - Jamaica Red Cross Society, 76 Arnold Road, Kingston 5.

JAPAN - Japanese Red Cross, 5 Shiba Park, Minato-Ku, Tokyo.

JORDAN - Jordan Red Crescent, P.O. Box 1337, Amman.

KENYA - Kenya Red Cross Society, St Johns Gate, P.O. Box 712, Nairobi.

KOREA (Democratic Republic) - Red Cross Society of the Democratic People's Republic of Korea, Pyongyang.

KOREA (Republic) - The Republic of Korea National Red Cross, 32-3 Ka Nam San-Donk, Seoul. 
LAOS - Laotian Red Cross, P.B. 46, Vientiane.

LEBANON - Lebanese Red Cross, rue Général Spears, Beirut.

LIBERIA - Liberian National Red Cross, National Headquarters, Corner of Tubman boulevard and 9th Street Sinkor, P.O. Box 226, Monrovia.

LIBYA - Libyan Red Crescent, Berka Omar Mukhtar Street, P.O. Box 541, Benghazi.

LIECHTENSTEIN - Liechtenstein Red Cross, Vadur.

LUXEMBURG - Luxemburg Red Cross, Parc de la Ville, C.P. 234, Luxemburg.

MADAGASCAR - Red Cross Society of Madagascar, rue Clemenceau, P.O. Box 1168 , Tananarive.

MALAYSIA - Malaysian Red Cross Society, 519 Jalan Belfield, Kuala Lumpur.

MALI - Mali Red Cross, B.P. 280, route de Koulikora, Bamako.

MEXICO - Mexican Red Cross, Sinaloa 20, $4^{\circ}$ piso, Mexico 7, D.F.

MONACO - Red Cross of Monaco, 27 Boul. de Suisse, Monte-Carlo.

MONGOLIA - Red Cross Society of the Mongolian People's Republic, Central Post Office, Post Box 537, Ulan-Bator.

MOROCCO - Moroccan Red Crescent, rue Calmette, B.P. 189, Rabat.

NEPAL - Nepal Red Cross Society, Tripureswore, P.B. 217, Kathmandu.

NETHERLANDS - Netherlands Red Cross, 27 Prinsessegracht, The Hague.

NEW ZEALAND - New Zealand Red Cross, 61 Dixon Street, P.O.B. 6073, Wellington C.2.

NICARAGUA - Nicaraguan Red Cross, 12 Avenida Noroeste, Managua, D.N.

NIGER - Red Cross Society of Niger, B.P. 386, Niamey.

NIGERIA - Nigerian Red Cross Society, Eko Akete Close, Ikoyi, Yaba, P.O. Box 764, Lagos.

NORWAY - Norwegian Red Cross, Parkveien 33b, Oslo.

PAKISTAN - Pakistan Red Cross, Frere Street, Karachi 4.

PANAMA - Panamanian Red Cross, Apartado 668, Panama.

PARAGUAY - Paraguayan Red Cross, calle André Barbero y Artigas 33, Asunción.

PERU - Peruvian Red Cross, Jiron Chancay 881, Lima.

PHILIPPINES - Philippine National Red Cross, 860 United Nations Avenue, P.O.B. 280, Manila.

POLAND - Polish Red Cross, Mokotowska 14, Warsaw.

PORTUGAL - Portuguese Red Cross, General Secretaryship, Jardim 9 de Abril, 1 a 5, Lisbon 3.

RUMANIA - Red Cross of the Rumanian Socialist Republic, Strada Biserica Amzei 29, Bucarest.

SALVADOR - Salvador Red Cross, 3a Avenida Norte y 3a Calle Poniente 21, San Salvador.
SAN MARINO - San Marino Red Cross, $\mathrm{Pa}$ gouvernemental, San Marino.

SAUDI ARABIA - Saudi Arabian Red Cresc Riyadh.

SENEGAL - Senegalese Red Cross Soci Bld. Franklin-Roosevelt, P.O.B. 299, Da

SIERRA LEONE - Sierra Leone Red C Society, 6 Liverpool Street, P.O.B. Freetown.

SOUTH AFRICA - South African Red $C_{1}$ Cor. Kruis \& Market Streets, P.O.B. 8 Johannesburg.

SPAIN - Spanish Red Cross, Eduardo Dato Madrid, 10.

SUDAN - Sudanese Red Crescent, P.O. 235, Khartoum.

SWEDEN - Swedish Red Cross, Artillerigata Stockholm 14.

SWITZERLAND - Swiss Red Cross, Taul strasse 8, B.P. 2699, 3001 Berne.

SYRIA - Syrian Red Crescent, 13, rue Abi-. Almaari, Damascus.

TANZANIA - Tanzania Red Cross Soci Upanga Road, P.O.B. 1133, Dar es Sala

THAILAND - Thai Red Cross Society, I Chulalongkorn Memorial Hospital, Bang

TOGO - Togolese Red Cross Society, Ave des Alliés 19, P.O. Box 655, Lome.

TRINIDAD AND TOBAGO - Trinidad Tobago Red Cross Society, 48 Pemb1 Street, P.O. Box 357, Port of Spain.

TUNISIA - Tunisian Red Crescent, 19 , d'Angleterre, Tunis.

TURKEY - Turkish Red Crescent, Yenise Ankara.

UGANDA - Uganda Red Cross, 17 Jinja R P.O. Box 494, Kampala.

UNITED ARAB REPUBLIC - Red Cres Society of the United Arab Republic, 34, Ramses, Cairo.

UPPER VOLTA - Upper Volta Red $C_{I}$ P.O.B. 340, Ouagadougou.

URUGUAY - Uruguayan Red Cross, Aveni de Octubre, 2990, Montevideo.

U.S.A. - American National Red Cross, and D Streets, N.W., Washington 6, D.C.

U.S.S.R. - Alliance of Red Cross and Crescent Societies, Tcheremushki, J. Tch mushkinskii proezd 5, Moscow W-36.

VENEZUELA - Venezuelan Red Cross, Ave Andrés Bello No. 4, Apart. 3185, Caracas.

VIET NAM (Democratic Republic) - Red C of the Democratic Republic of Viet $\mathrm{N}$ 68, rue Bà-Trièz, Hanoi.

VIET NAM (Republic) - Red Cross of Republic of Viet Nam, 201, duong Hi Thâp-Tu, No. 201, Saigon.

YUGOSLAVIA - Yugoslav Red Cross, Sin ulica broj 19, Belgrade.

ZAMBIA - Zambia Red Cross, P.O. R. W. 1, Ridgeway, Lusaka. 